\title{
Familial Mediterranean Fever Mimicking Wilson's Disease: A Case Report
}

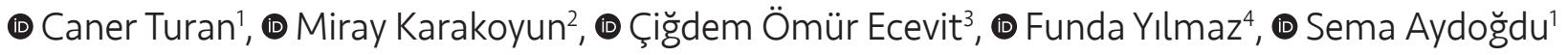 \\ 'Ege University Faculty of Medicine, Department of Pediatric Gastroenterology, İzmir, Turkey \\ 2Universityof Health Sciences, İzmir Tepecik Training and Research Hospital, Clinic of Pediatric Gastroenterology, İzmir, Turkey \\ ${ }^{3}$ University of Health Sciences, İzmir Dr. Behçet Uz Children Diseases and Surgery Training and Research Hospital, Clinic of Pediatric Gastroenterology, \\ İmir, Turkey \\ ${ }^{4}$ Ege University Faculty of Medicine, Department of Pathology, İzmir, Turkey
}

\begin{abstract}
Wilson's disease (hepatolenticular degeneration) is an autosomal recessive defect in cellular copper transport. Impaired biliary copper excretion leads to an accumulation of copper mostly in the liver, brain and cornea. Familial Mediterranean Fever (FMF) is an autosomal recessive autoimmune disease as a result of a mutation in the MEFV gene encoding pyrin protein characterized by recurring fever and polyserositis attacks. In this report, we describe a Turkish female child with cholestatic hepatitis of unknown etiology who was later diagnosed with typical FMF.

Keywords: Familial Mediterranean Fever, Wilson's disease, liver, cryptogenic cirrhosis, cholestasis
\end{abstract}

\section{Introduction}

Wilson's disease (hepatolenticular degeneration) is an autosomal recessive defect in cellular copper transport. Impaired biliary copper excretion leads to an accumulation of copper mostly in the liver, brain and cornea. Different clinical manifestations of hepatic copper accumulation (acute hepatitis, chronic hepatitis, acute liver failure, cirrhosis) have been seen in Wilson's disease $(1,2)$. The autosomal recessive disease Familial Mediterranean Fever (FMF), as the name suggests, is predominantly found in the Mediterranean populations; this includes Turks, Arabs, Armenians and non-Ashkenazi Jews. The disease is characterized by febrile, recurrent inflammatory attacks of the serosal membranes, causing a prolonged self-limitation (3). FMF has been linked to liver failure if left untreated over an extended period; this connection, however, has rarely been reported. In this report, we describe a patient admitted with acute cholestatic hepatitis who was later diagnosed with typical FMF.

\section{Case Report}

A 7-year-old female presented with jaundice, 2-3 reported instances of yellow-coloured stools and pruritus over a 5-day period. She had had abdominal pain, vomiting and poor feeding during the previous two days. The patient was seen regularly for health maintenance visits and she was generally a healthy child. She was up to date with her immunizations at that time. Verbal consent was taken from the patient's parents. Physical examination indicated poor overall health. However, her growth and development were considered normal. Her spleen and liver were enlarged with inferior margins of $4 \mathrm{~cm}$ and 5 $\mathrm{cm}$ respectively under the costal line. Blood analysis results revealed haemoglobin of $11.3 \mathrm{~g} / \mathrm{dL}$, white blood cell count of

\section{Address for Correspondence}

Caner Turan MD, Ege University Faculty of Medicine, Department of Pediatric Gastroenterology, izmir, Turkey Phone: +90 5554153900 E-mail: canertrn@yahoo.com ORCID ID: orcid.org/0000-0001-9469-5162 Received: 06.04 .2017 Accepted: 23.05.2017

${ }^{\circ}$ Copyright 2018 by Ege University Faculty of Medicine, Department of Pediatrics and Ege Children's Foundation The Journal of Pediatric Research, published by Galenos Publishing House. 
$14.100 / \mathrm{mm}^{3}$, with $72 \%$ neutrophils and a platelet count of $703.000 / \mathrm{mm}^{3}$. The erythrocyte sedimentation rate (ESR) was elevated at $32 \mathrm{~mm} / \mathrm{h}$. C-reactive protein (CRP) was also elevated at $3.3 \mathrm{mg} / \mathrm{dL}$. Prothrombin time, partial thromboplastin time and fibrinogen values were markedly abnormal. Liver function tests revealed elevated levels of aspartate aminotransferase $(1.835 \mathrm{U} / \mathrm{L})$, alanine aminotransferase $(1.114 \mathrm{U} / \mathrm{L})$, lactate dehydrogenase level $(698 \mathrm{U} / \mathrm{L}), \gamma$-glutamyl transferase (128 $\mathrm{U} / \mathrm{L})$, total bilirubin $(20.45 \mathrm{mg} / \mathrm{dL})$ and conjugated bilirubin (10.8 mg/dL). Her condition was evaluated as acute hepatitis with hyperbilirubinemia, coagulopathy and not accompanied encephalopathy (fulminant hepatitis). Antibody screening for hepatitis A, hepatitis B and hepatitis C viruses and for Epstein-Barr virus were negative. Toxoplasmosis was negative. Cytomegalovirus (CMV) antigen and CMV DNA were also negative. Tests for anti-liver-kidney microsomal anti-bodies Type I, anti-soluble liver antigen, anti-liver-pancreas antigen, anti-smooth muscle antibodies and anti-nuclear antibodies were all negative. Alpha-1 antitrypsin and sweat test were normal. The serum level of ceruloplasmin was $24 \mathrm{mg} / \mathrm{dL}$ (normal $>20 \mathrm{mg} / \mathrm{dL}$ ) and non-ceruloplasmin-bound copper level was elevated at $38 \mathrm{mcg} / \mathrm{dL}$ (normal $<15 \mathrm{mcg} / \mathrm{dL}$ ). The urinary copper level was $387 \mathrm{mcg} / 24 \mathrm{~h}$ and $128 \mathrm{mcg} / 24 \mathrm{~h}$ (normal $\leq 30$ to $40 \mathrm{mcg} / 24 \mathrm{~h}$ ). Her neurological examination was normal. The patient was administered a combination treatment of $\mathrm{N}$-acetylcysteine (Asist 10\%, Hüsnü Arsan Medical, İstanbul, Turkey) $(5 \mathrm{mg} / \mathrm{kg} / \mathrm{h})$, ursodeoxycholic acid (Ursofalk, Aris Medical, İstanbul, Turkey) (15 mg/kg/d), zinc (Zinco-220, Berko Medical, İstanbul, Turkey) $(2 \mathrm{mg} / \mathrm{kg} / \mathrm{d})$ and ampicillin (Ampisina, Mustafa Nevzat Medical, İstanbul, Turkey) due to the indication of liver insufficiency with unknown etiology. A liver biopsy was also performed. At the one-week follow-up, regarding the examination of the liver biopsy, all coagulation markers and transaminase levels had returned to normal levels quickly with the exception of cholestatic hepatitis. This quick decline of transaminase levels was attributed to the zinc supplementation. In spite of her normal ceruloplasmin levels, Wilson's disease was originally considered due to elevated urinary copper levels. D-penicillamin (Metalcaptase TBL, Actavis Medical, Czech Republic) treatment (250 mg) was initiated; increased to $500 \mathrm{mg}$ after 1 week. A histological examination of the liver biopsy specimens showed moderate fibrosis with portal inflammations (Figure 1-3). Copper and orcein staining were negative and the dry liver copper weight was $21.8 \mathrm{mcg} / \mathrm{g}$. The abnormal liver function improved and the patient was discharged. During the follow-up period, liver function tests were normal, however, an enlarged liver and spleen with elevated ESR and CRP levels were still being noted. A very detailed history of the patient revealed that she had been having attacks of arthralgia with fever three to four times per year, which now made FMF a strong possibility. At that time, a molecular analysis for Wilson disease's proved negative and informed consent was obtained in order to perform a

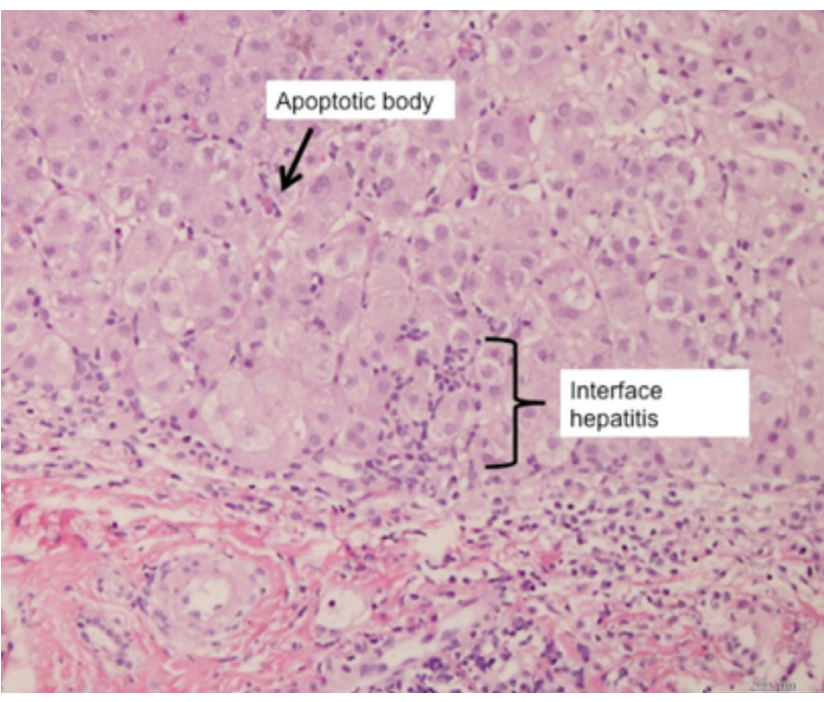

Figure 1. Interface hepatitis and apoptotic body (hematoxylin and eosin, x20)

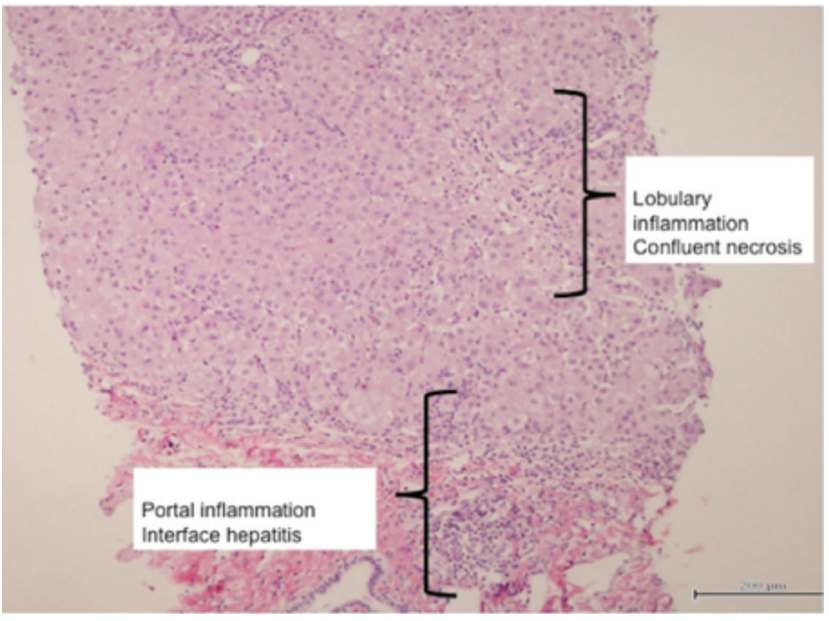

Figure 2. Lobulary inflammation, portal inflammation and confluent necrosis (hematoxylin and eosin, x10)

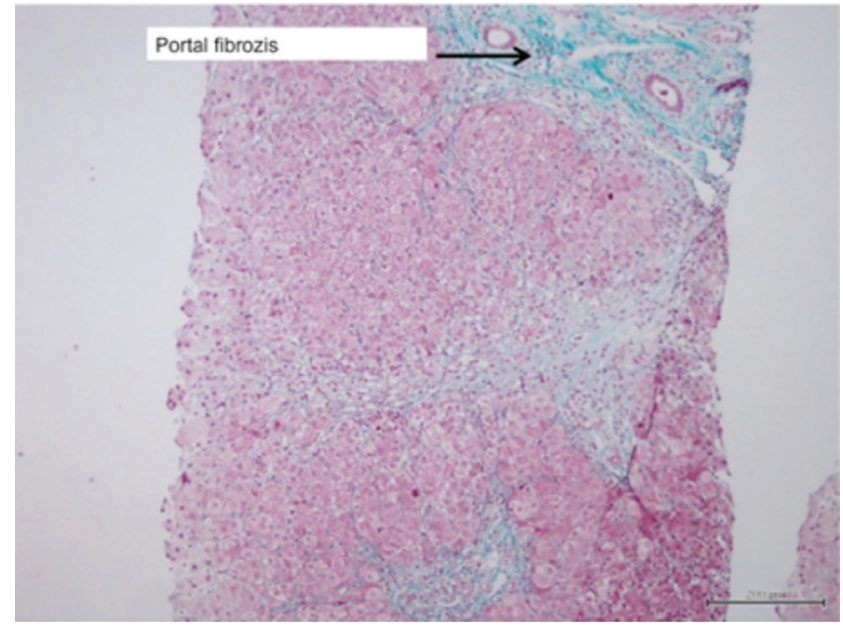

Figure 3. Portal fibrosis (hematoxylin and eosin, x10) 
genotype analysis. After informed consent was obtained, genomic DNA was then extracted from the peripheral blood cells. Results showed a homozygous M694V mutation. Colchicine therapy at an average daily dose of $1.0 \mathrm{mg} /$ day was initiated immediately, with d-penicillamin treatment being decreased. The patient responded to colchicine treatment and d-penicillamin treatment was completely discontinued.

The diagnosis of FMF was given after the history of arthralgia and fever attacks ( $\geq 3$ attacks per year) became known, the homozygous M694V mutation was detected and there was a response to the colchicine therapy. Therefore, she presented two major and one minor criteria of FMF and the genetic mutation of FMF.

A follow-up examination one year later showed the patient had no clinical signs and all laboratory tests were within the normal ranges.

\section{Discussion}

The most prominent characteristics of FMF are short, acute attacks of abdominal pain and fever accompanied by serosal, synovial and cutaneous inflammation. While the liver is one of the most affected organs seen in FMF disease, Nonamyloid liver disease concomitant with FMF has rarely been reported. Korkmaz and Kaşifoğlu (4) reported mild hyperbilirubinemia in 11 of 41 adult patients and elevated transaminases levels in $9.7 \%$ percent of patients during acute FMF attacks. Migita et al. (5) described one female patient with hepatitis who was later diagnosed as typical FMF. In another study, Rimar et al. (6) reported an association between FMF and non-alcoholic steatohepatitis. Seventyfour percent of their FMF patients had originally been referred for assessment due to chronic liver disease. In the literature, however, only limited case reports involving recurrent acute cryptogenic hepatitis are available (7). Sari et al., (8) reported two cases with Budd-Chiari syndrome associated with FMF. They were characterized by a hypercoagulable state or ongoing inflammation that activated coagulation through endothelial damage, possibly leading to thrombosis. Unal et al. (9) found liver involvement in 11 of 58 pediatric FMF cases. One of their patients, originally admitted with acute cholestatic hepatitis and later diagnosed with FMF, was phenotypically similar to our patient in this case report. The molecular analysis of our patient for FMF revealed a homozygous M694V mutation. Tweezer-Zaks et al. (10) had previously suggested the possible association between FMF and homozygous M694V MEFV mutation with cryptogenic cirrhosis. They speculated that mutated MEFV may play a modifier role in cryptogenic cirrhosis. Unal et al. (9) determined that, while M694V allele mutations were a strong indicator of FMF, mutations without the underlying clinical manifestations had been observed. Future studies analyzing MEFV gene sequences in a large cohort of patients with liver involvement are needed to truly verify this possibility. This case study emphasizes the fact that FMF may present with cryptogenic hepatitis during the early years of life. Clinicians in Mediterranean countries (such as Turkey) where the prevalence of FMF is high must consider this when making their differential diagnosis.

\section{Acknowledgements}

We would like to thank the family of the patient for participating in the study. Also, the authors would like to forward sincere thanks to the large team who worked together including technical help, writing assistance and the departmental head who all provided general support.

\section{Ethics}

Informed Consent: The verbal consent was taken from the patient's parents.

Peer-review: External and internal peer-reviewed.

\section{Authorship Contributions}

Surgical and Medical Practices: C.T., M.K., Concept: C.T., M.K., S.A., Design: C.T., M.K., Ç.Ö.E., Data Collection and Processing: C.T., Ç.Ö.E., F.Y., Analysis and Interpretation: C.T., Ç.Ö.E., S.A., Literature Search: C.T., Ç.Ö.E., Writing: C.T., Ç.Ö.E., S.A.

Conflict of Interest: No conflict of interest was declared by the authors.

Financial Disclosure: The authors declared that this study received no financial support.

\section{References}

1. Steindl P, Ferenci P, Dienes HP, et al. Wilson's disease in patients presenting with liver disease: a diagnostic challenge. Gastroenterology 1997; 113:212-8.

2. Ferenci P. Pathophysiology and clinical features of Wilson disease. Metab Brain Dis 2004; 19:229-39.

3. Ben-Chetrit E, Levy M. Familial Mediterranean fever. Lancet 1998; 351:659-63.

4. Korkmaz C, Kaşifoğlu T. Changes in the liver function tests during the attacks of familial Mediterranean fever. Rheumatol Int 2007; 27:395-8.

5. Migita K, Abiru S, Tanaka M, et al. Acute hepatitis in a patient with familial Mediterranean fever. Liver Int 2008; 28:140-2.

6. Rimar D, Rosner I, Rozenbaum M, Zuckerman E. Familial Mediterranean fever: an association with non-alcoholic fatty liver disease. Clin Rheumatol 2011; 30:987-91.

7. Neequaye J, Jelly AE. Acute hepatitis in recurrent hereditary polyserositis (familial Mediterranean fever). I Trop Pediatr 1994; 40:243-5.

8. Sari S, Egritas O, Bukulmez A, Dalgic B, Soylemezoglu O. Is familial Mediterranean fever a possible cofactor for Budd-Chiari syndrome? J Pediatr Gastroenterol Nutr 2009; 49:481-4.

9. Unal $F$, Cakir $M$, Baran $M$, et al. Liver involvement in children with Familial Mediterranean fever. Dig Liver Dis 2012; 44:689-93.

10. Tweezer-Zaks N, Doron-Libner A, Weiss P, et al. Familial Mediterranean fever and cryptogenic cirrhosis. Medicine 2007; 86:355-62. 